\title{
Relación entre la capacidad cardiorrespiratoria y el rendimiento en los tests de condición física relacionada con la salud incluidos en la batería ALPHA en niños de 10-12 años
}

\author{
Relationship between cardiorespiratory fitness and performance in the ALPHA \\ health-related physical fitness test battery for 10-12 year-old children
}

\author{
Daniel Mayorga-Vega', Rafael Merino-Marban², Emilio Rodríguez-Fernández ${ }^{2}$ \\ 1 Departamento de Educación Física y Deportiva, Universidad de Granada, España \\ 2 Facultad de Ciencias de la Educación, Universidad de Málaga, España
}

\section{Resumen}

La condición física es considerada un importante marcador relacionado con la salud en la infancia. Entre sus diferentes componentes, la resistencia cardiorrespiratoria ha sido la que constituye el principal exponente de salud. Sin embargo, su relación con otros componentes de la condición física aún no ha sido ampliamente estudiada entre los niños. El objetivo del presente estudio fue analizar la relación de presentar una alta (o baja) capacidad cardiorrespiratoria y el nivel de condición física relacionada con la salud en niños de 10-12 años. Una muestra de 72 escolares (niños, $\mathrm{n}=37$; niñas, $\mathrm{n}=$ 35) voluntarios de 10-12 años de edad participaron en el presente estudio. Los estudiantes fueron evaluados mediante la batería ALPHA basada en la evidencia durante dos clases de Educación Física. En la primera sesión se realizaron las medidas de composición corporal (peso, talla y pliegues cutáneos) y en la segunda las pruebas de dinamometría manual, salto de longitud y Course Navette. Los niños que presentaban una alta condición física cardiovascular ( $\geq$ percentil 50 en las marcas del test Course Navette) mostraron menores valores de IMC, pliegues cutáneos, porcentaje de grasa corporal, así como mayores valores de salto de longitud, Course Navette y consumo máximo de oxígeno estimado que los del grupo de baja condición física cardiovascular (< percentil 50 en las marcas del test Course Navette). Las pruebas incluidas en la batería de tests ALPHA basada en la evidencia parecen presentar una clara relación con la salud cardiovascular de los niños de 10-12 años.

Palabras clave: Capacidad cardiovascular, VO2max, fuerza muscular, composición corporal, escolares, jóvenes.

\section{Abstract}

Physical fitness is considered an important healthrelated marker during childhood. Among its various components, cardiorespiratory fitness constitutes the leading component of health. However, its relationship with other physical fitness components has not been widely studied among children. The purpose of this study was to examine the relationship of having high (or low) cardiorespiratory fitness and health-related physical fitness in children 10 to 12 years of age. A sample of 72 10-12 year-old students (boys, $n=37$; girls, $n=35$ ) volunteered to participate in this study. During two sessions of Physical Education, students were evaluated by the evidence-based ALPHA healthrelated fitness test battery. During the first session, body composition measures (weight, height and skinfolds) were taken, and in the second session handgrip strength, standing long jump, and a 20-m shuttle run test were performed. Children with high cardiovascular fitness ( $\geq 50$ th percentile in shuttle run test scores) showed lower values for BMI, skinfolds, and body fat percentage and higher values for standing long jump, the shuttle run test, and maximal oxygen consumption than the low cardiovascular fitness group ( $<50$ th percentile in shuttle run test scores). The tests contained in the evidence-based ALPHA test battery seem to have a clear relationship with the cardiovascular health of 10-to-12-year-old children.

Key words: Cardiovascular fitness, VO2max, muscular strength, body composition, schoolchildren, youth. 


\section{Introducción}

La condición física puede ser considerada como una medida integrada de las principales funciones del cuerpo (musculo-esquelética, cardio-respiratoria, hematocirculatoria, endocrino-metabólica y psico-neurológica) que participan en la realización de actividad física (Ortega, Ruiz, Castillo y Sjöström, 2008). Por lo tanto, la evaluación de la condición física constituye una medida integrada de todas estas funciones. Un alto nivel de condición física implica una buena respuesta fisiológica. Por el contrario, tener un bajo nivel de condición física podría indicar un mal funcionamiento de una o varias de esas funciones (Ardoy et al., 2010).

Recientes investigaciones con adultos han puesto de manifiesto el interés que tiene conocer el estado de la condición física que posee una persona, ya que constituye un excelente predictor de la esperanza y calidad de vida (Myers et al., 2002). Entre los diferentes componentes, la resistencia cardiorrespiratoria ha sido la que constituye el principal exponente de la condición física del individuo. Importantes estudios prospectivos han demostrado de manera inequívoca que la capacidad cardiorrespiratoria es el predictor más potente de mortalidad y morbilidad (Kodama et al., 2009). De hecho, se ha demostrado que un bajo nivel de condición física cardiovascular constituye el factor de riesgo cardiovascular más importante, por encima de factores de riesgo clásicos como, por ejemplo, el sobrepeso u obesidad (Castillo Garzón, Ortega Porcel, y Ruiz Ruiz, 2005).

Además, se ha visto también que existe una relación inversa entre capacidad cardiorrespiratoria ( $\mathrm{VO}_{2} \mathrm{max}$ ) y mortalidad por cáncer, independientemente de la edad, hábito alcohólico, padecimiento de diabetes mellitus e incluso consumo de tabaco (Evenson, Stevens, Cai, Thomas, y Thomas, 2003; Lee y Blair, 2002; Sawada et al., 2003b). Del mismo modo, se ha constatado que el $\mathrm{VO}_{2}$ max es un importante determinante de la sensibilidad a la insulina (Sawada, Lee, Muto, Matuszaki, y Blair, 2003a; Seibaek et al., 2003), y bajos niveles del mismo se asocian con el padecimiento del denominado "síndrome metabólico" (obesidad abdominal, intolerancia a la glucosa, diabetes tipo II, hipertensión, hiperlipidemia y resistencia a la insulina) (Bertoli et al., 2003; Lakka et al., 2003).

La infancia es una etapa crucial de la vida, ya que los drásticos cambios fisiológicos y psicológicos tienen lugar en estas edades. Del mismo modo, los hábitos de vida se establecen durante estos años, lo que podría influir en el comportamiento en la vida adulta y el estado de salud (Ortega et al., 2008). A pesar de que los estudios con niños en los que se relaciona la condición física con la salud no son demasiados (Froberg y Andersen, 2005), existen datos nacionales que demuestran que un bajo grado de condición física cardiovascular en la adolescencia se relaciona de forma directa con un riesgo aumentado de padecer enfermedades cardiovasculares en edades más avanzadas (GonzálezGross et al., 2003; Ortega et al., 2005).

La preocupación por la condición física relacionada con la salud de nuestros escolares ha aumentado enormemente (de la Cruz Sánchez y Pino Ortega, 2010; Salinas Martínez, Miranda León y Viciana Ramírez, 2006), especialmente en la mejora de la condición física cardiovascular (Navarro et al., 2010; RamírezLechuga et al., 2010). Varios estudios han encontrado una asociación entre los niveles de capacidad cardiorrespiratoria y otros componentes de la condición física como la composición corporal, fuerza muscular, flexibilidad, velocidad-agilidad y coordinación (Ara, Moreno, Leiva, Gutin, y Casajús, 2007; Brunet, Chaput, y Tremblay, 2007). Sin embargo, la relación entre un alto (o bajo) nivel de capacidad cardiorrespiratoria y otros parámetros de la condición física aún no ha sido ampliamente estudiada entre los jóvenes (Ortega et al., 2005; Casajús et al., 2006), no habiéndose encontrado estudios exclusivamente con niños. Consecuentemente, el objetivo del presente estudio fue analizar la relación de presentar una alta (o baja) capacidad cardiorrespiratoria y los niveles de condición física relacionada con la salud en niños y niñas de 10 a 12 años.

\section{Método}

\section{Participantes}

Una muestra de 72 escolares voluntarios de 10-12 años de edad participaron en el presente estudio. Los participantes fueron niños $(n=37)$ y niñas $(n=35)$ aparentemente sanos de $5^{\circ}$ y $6^{\circ}$ curso de Educación Primaria de un centro escolar de la provincia de Málaga (España). Los criterios de inclusión seguidos fueron: a) tener una edad comprendida entre los 10 y 12 años; b) no presentar aparentes restricciones de salud que pudieran limitar la ejecución de las pruebas; c) no haber realizado actividad física intensa en las 48 horas previas a la evaluación.

Se dio una descripción completa de la naturaleza y el propósito del estudio a los niños, padres, director y profesores. Se obtuvo el consentimiento informado por parte del director del centro y de los padres de los escolares. El estudio fue aprobado por el Comité de Ética de la Universidad de Málaga. Además, el estudio fue realizado de acuerdo con los procedimientos esta- 
blecidos en la Declaración de Helsinki de la Asociación Médica Mundial (2008) sobre principios éticos para las investigaciones médicas en seres humanos.

\section{Procedimiento}

Antes del estudio se realizó una prueba piloto para valorar la fiabilidad de las medidas administradas. Los niños realizaron los test incluidos en la batería de pruebas de condición física relacionada con la salud ALPHA basada en la evidencia (Ruiz et al., 2011) por incluir todas las medidas que presentan una fuerte relación con el estado de salud actual y futuro para los niños (Ortega et al., 2008). Sin embargo, por cuestiones relacionadas con la intimidad y el pudor al tratarse de menores los perímetros de la cintura no fueron tomados. Por las mismas razones, tomamos los pliegues cutáneos del tríceps y la pierna medial (en vez de los pliegues del tríceps y subescapular como se propone en la antedicha batería) para la estimación de la grasa corporal entre los niños (Alvero Cruz et al., 2009).

Para su aplicación dos evaluadores experimentados administraron las pruebas siguiendo el protocolo establecido. Cada evaluador realizó la misma prueba a todos los alumnos. Durante la primera sesión se realizaron las medidas de composición corporal en el siguiente orden: peso, talla, y pliegues cutáneos. En la siguiente sesión se realizaron las pruebas de dinamometría manual, salto de longitud y Course Navette. La evaluación de los estudiantes se realizó durante las clases de Educación Física. Todas las pruebas fueron administradas durante la misma semana, el mismo periodo del día y bajo las mismas condiciones medioambientales para cada estudiante. Previo a la segunda sesión todos los participantes realizaron un calentamiento estandarizado de cinco minutos de carrera a baja intensidad. Durante la sesión de composición corporal no se realizó ningún calentamiento.

1. Índice de Masa Corporal (IMC). Para evaluar el IMC previamente se obtuvo el peso y la talla de todos los estudiantes. Para el peso el participante permaneció de pie en el centro de la plataforma (SECA, LTD, Alemania) llevando una ropa ligera (excluyendo zapatos, pantalones largos y sudaderas) y con el peso distribuido por igual en ambos pies y sin apoyos. Para medir la altura el participante permaneció de pie, con los talones juntos, brazos a lo largo del cuerpo y nalgas y espalda apoyadas sobre la escala (Holtain Ltd., Dyfed, UK) con la cabeza colocada en el plano de Frankfort. Las medias de los dos intentos del peso y la talla fueron utilizadas para el posterior análisis estadístico. La fiabilidad estimada de la medidas fueron: peso, CCI
(IC) $=0,99(0,99-1,00)$, SEM = 1,21 kg; talla, CCI (IC) $=0,99(0,99-1,00), \mathrm{SEM}=0,90 \mathrm{~cm}$. El IMC fue calculado como el peso en kilogramos dividido por la talla en metros al cuadrado $\left(\mathrm{kg} / \mathrm{m}^{2}\right)$.

2. Pliegues cutáneos (triceps + pierna medial). Los pliegues cutáneos fueron tomados para determinar la amplitud del pliegue cutáneo y así poder estimar el porcentaje de grasa corporal. En primer lugar, para la marca del pliegue del tríceps se señaló con un lápiz dermográfico el punto más lateral y superior del acromion. Luego se marcó el borde proximal de la cabeza del radio. Con la cinta antropométrica se determinó el punto medio entre la referencia acromial y radial. Por último, esta línea fue prolongada por la cara posterior del brazo, la cual era cortada perpendicularmente por una línea desde el olecranon. En segundo lugar, para la marca del pliegue de la pierna medial se marcó la intersección de la cara medial y el máximo perímetro de la pierna (International Society for the Advancement of Kinanthropometry [ISAK], 2001).

Para la toma del pliegue del tríceps el niño se situó de pie con el hombro derecho en ligera rotación externa y el codo extendido a lo largo del cuerpo. Para la toma del pliegue de la pierna medial el niño se situó de pie con la pierna derecha sobre un banco con la rodilla a $90^{\circ}$. Mientras se tomaba el pliegue sobre la marca con el dedo pulgar e índice de la mano izquierda, se midió con un plicómetro en la mano derecha a un centímetro inferior de los dedos. En ambos casos se realizó la medida sobre las extremidades derechas. La lectura de la marca fue realizada a los dos segundos. Se realizaron dos mediciones no consecutivas de ambos pliegues tomando la media de las dos medidas para el posterior análisis estadístico (ISAK, 2001). La fiabilidad estimada de la medidas fueron: tríceps, CCI (IC) = 0,99 (0,99-1,00), SEM = 0,53 mm; pierna medial, CCI $(I C)=0,99(0,99-1,00), S E M=0,60 \mathrm{~mm}$. Para estimar el porcentaje de masa grasa se usaron las ecuaciones propuestas por Slaughter et al. (1988).

3. Dinamometría manual. Previamente a la realización del test el evaluador medía el tamaño de la mano derecha del evaluado usando la tabla-regla para ver la envergadura de agarre óptima de acuerdo al tamaño de su mano (España-Romero et al., 2008). El tamaño de la mano se midió como la máxima distancia de separación entre el primer y quinto dedo. La fuerza de presión manual fue medida usando un dinamómetro digital (T.K.K. 5101 Grip-D; Takey, Tokyo, Japan) y los valores fueron recogidos en kilogramos. El niño se situaba de pie con los brazos a lo largo del cuerpo con los hombros ligeramente abducidos $\left(<10^{\circ}\right)$, el codo extendido y el antebrazo y la muñeca en una posición neutra (España-Romero et al., 2008). 
Durante la realización del test, el niño era instruido para mantener la posición estándar de bipedestación con el codo en completa extensión y sin tocar ninguna parte del cuerpo con el dinamómetro (Ruiz et al., 2006). Cada niño realizó el test dos veces (alternativamente con ambas manos) en orden aleatorio, con un minuto de descanso entre repeticiones (Watanabe et al., 2005). Para cada medida, la mano evaluada en primer lugar fue elegida aleatoriamente. La duración del test fue de $5 \mathrm{~s}$ por intento. El evaluador situaba el marcador a cero después de cada intento. El mejor intento de cada mano fue usado para el posterior análisis estadístico. La fiabilidad estimada de la medidas fueron: mano derecha, $\mathrm{CCI}(\mathrm{IC})=0,93(0,89-0,96), \mathrm{SEM}=$ $1,14 \mathrm{~kg}$; mano izquierda, CCI (IC) = 0,95 (0,91-0,97), $\mathrm{SEM}=1,02 \mathrm{~kg}$.

4. Salto de longitud. La prueba se realizó sobre una superficie horizontal y antideslizante. Una cinta métrica fue extendida en el suelo para medir la distancia del salto. Perpendicularmente a la marca del $0 \mathrm{~cm}$ se colocaba una marca en el suelo donde los niños debían situar las puntas de los pies. El investigador mostró la correcta ejecución previamente a su realización. Luego a cada niño se le permitió un ensayo antes de la prueba. Desde una posición de pie, con los pies a la anchura de los hombros, el niño realizó un contra-movimiento con las piernas antes de realizar un salto horizontal lo más lejos posible.

Durante el contra-movimiento, los niños usaron sus brazos para ayudarse en el salto. Cuando el niño aterrizaba, debía mantener los pies fijos en el suelo para su medición. Si el niño tocaba con alguna parte del cuerpo la zona que quedaba detrás de los pies, el intento era nulo y se repetía de nuevo. Sin embargo, el niño podía colocar las manos por delante para ayudarse en el aterrizaje. El investigador evaluó la distancia de la parte posterior del pie más retrasada con la ayuda de un cartabón que situaba a lo largo de la cinta métrica. Los niños realizaron dos intentos con un minuto de recuperación. La mejor marca fue registrada para el posterior análisis estadístico. La fiabilidad estimada para la medida del salto de longitud fue: $\mathrm{CCI}$ (IC) = 0,94 (0,90-0,96), SEM $=5,36 \mathrm{~cm}$.

5. Course Navette. Todos los estudiantes corrieron entre dos líneas paralelas separadas por $20 \mathrm{~m}$ de distancia al ritmo marcado por una señal emitida por la grabación. Cada niño se situaba a $1 \mathrm{~m}$ de distancia de los compañeros contiguos. Se utilizaron las líneas marcadas en la propia pista, colocándose un cono en cada una de las esquinas para delimitar mejor la zona de carrera durante la prueba. Para ayudar a marcar el ritmo de carrera un investigador realizó el test junto a los niños. La velocidad inicial era de $8,5 \mathrm{~km} / \mathrm{h}$, la cual fue incrementada $0,5 \mathrm{~km} / \mathrm{h}$ cada minuto (un minuto representa un palier). Los participantes fueron instruidos para correr en línea recta cruzando la línea con ambos pies. Los niños debían salir de la línea inmediatamente después de escuchar cada señal.

El test acababa cuando el niño se detenía debido a la fatiga o cuando no alcanzaba la línea antes de la siguiente señal en dos veces consecutivas. Los niños fueron constantemente alentados a correr durante el transcurso de la carrera. Cada niño realizó un solo intento. El último palier completado fue registrado para el posterior análisis estadístico. La fiabilidad estimada para la medida del Course Navette fue: CCI (IC) = 0,90 $(0,81-0,95), \operatorname{SEM}=0,61$ paliers. Se usó la ecuación de Léger, Mercier, Gadoury, y Lambert (1988) para estimar el consumo máximo de oxígeno.

\section{Análisis estadístico}

Se realizó una estadística descriptiva (media \pm desviación estándar) de la edad, peso, talla, IMC, pliegues del tríceps y pierna medial, porcentaje de grasa corporal, dinamometría manual, salto de longitud, Course Navette y $\mathrm{VO}_{2}$ máx. Para comprobar la fiabilidad de las medidas empleadas se utilizó el coeficiente de correlación intraclase $\left(\mathrm{CCI}_{3, k}\right)$, acompañado del intervalo de confianza al 95\% (IC), y el error estándar de la medida (SEM) (Weir, 2005). Se aplicó la prueba análisis de la varianza (ANOVA) de un factor para analizar las posibles diferencias en la media de los valores de las medidas tomadas entre los alumnos con baja condición física cardiovascular (BCF) y alta condición física cardiovascular (ACF). Para ello, previamente los alumnos se dividieron en base a los valores obtenidos en el test Course Navette ( $\mathrm{n}^{\circ}$ de vueltas totales) en: BCF < percentil 50 y $\mathrm{ACF} \geq$ percentil 50 . Además, se calculó la eta al cuadrado $\left(\eta^{2}\right)$ para estimar la magnitud de la diferencia. Por último, se utilizó la prueba chi-cuadrado para analizar las diferencias en la frecuencia de género entre los dos grupos. El análisis estadístico fue realizado mediante el paquete estadístico SPSS 15.0 para Windows (SPSS ${ }^{\circledR}$ Inc., Chicago, IL). El nivel de significación se estableció en $\mathrm{p}<0,05$.

\section{Resultados}

En la Tabla 1 se encuentra la media y deviación estándar de los valores obtenidos en los diferentes tests de condición física para los niños con BCF y ACF. Los resultados del ANOVA de un factor mostraron valores estadísticamente significativas mayores $(p<0,001)$ 
Tabla 1. Resultados del ANOVA de un factor entre los alumnos con baja condición física cardiovascular y alta condición física cardiovascular.

\begin{tabular}{|c|c|c|c|c|c|}
\hline Medidas & $B C F(n=35)$ & $\operatorname{ACF}(n=37)$ & $\mathbf{F}$ & $\mathrm{p}$ & $\eta^{2}$ \\
\hline Género (niños/ niñas) & $13 / 22$ & $24 / 13$ & - & - & - \\
\hline Edad (años) & $10,89 \pm 0,68$ & $10,92 \pm 0,68$ & 0,043 & 0,836 & 0,001 \\
\hline Peso $(\mathrm{kg})$ & $52,41 \pm 12,31$ & $42,41 \pm 9,81$ & 14,649 & $<0,001$ & 0,173 \\
\hline Talla $(\mathrm{cm})$ & $149,43 \pm 9,25$ & $147,76 \pm 8,77$ & 0,620 & 0,434 & 0,009 \\
\hline $\mathrm{IMC}\left(\mathrm{kg} / \mathrm{m}^{2}\right)$ & $23,23 \pm 3,76$ & $19,23 \pm 2,97$ & 25,193 & $<0,001$ & 0,265 \\
\hline Pliegue triceps (mm) & $24,74 \pm 6,33$ & $18,46 \pm 5,91$ & 18,959 & $<0,001$ & 0,213 \\
\hline Pliegue pierna medial (mm) & $30,03 \pm 6,83$ & $21,91 \pm 6,35$ & 27,370 & $<0,001$ & 0,281 \\
\hline Grasa corporal (\%) & $39,65 \pm 8,78$ & $30,23 \pm 8,35$ & 21,766 & $<0,001$ & 0,237 \\
\hline Dinamometría manuala (kg) & $18,09 \pm 3,54$ & $19,57 \pm 4,81$ & 2,166 & 0,143 & 0,030 \\
\hline Salto de longitud $(\mathrm{cm})$ & $124,06 \pm 16,32$ & $152,19 \pm 16,78$ & 51,123 & $<0,001$ & 0,426 \\
\hline Course Navette (vueltas) & $20,97 \pm 7,45$ & $47,27 \pm 11,72$ & 127,451 & $<0,001$ & 0,646 \\
\hline $\mathrm{VO}_{2} \operatorname{máx}(\mathrm{ml} / / \mathrm{kg} / \mathrm{min})$ & $40,36 \pm 2,26$ & $48,16 \pm 3,28$ & 136,343 & $<0,001$ & 0,661 \\
\hline
\end{tabular}

Nota. Los datos son presentados como la media \pm desviación estándar, excepto para el género que muestra la frecuencia de niños/ niñas; BCF = Baja condición física cardiovascular, ACF = Alta condición física cardiovascular, IMC = Índice de masa corporal, VO2máx = Consumo de oxígeno máximo estimado; En la tabla se muestra la media de los valores del lado derecho e izquierdo.

para los participantes de BCF en el peso, IMC, pliegue del tríceps y la pierna medial, y porcentaje estimado de grasa corporal. Los estudiantes del grupo con ACF presentaron mayores valores $(p<0,001)$ en las pruebas de salto de longitud, el Course Navette y el consumo de oxígeno máximo estimado. En la edad, la talla y la fuerza medida mediante la dinamometría manual no se encontraron diferencias estadísticamente significativas entre ambos grupos ( $\mathrm{p}>0,05)$. Por último, la prueba chi-cuadrado mostró que el grupo con $\mathrm{BCF}$ presentaba una mayor proporción de niñas y el de ACF una mayor proporción de niños $\left(\chi_{1}^{2}=5,533 ; \mathrm{p}=0,019\right)$.

\section{Discusión}

El objetivo del presente estudio fue analizar la relación de presentar una alta (o baja) capacidad cardiorrespiratoria y los niveles de condición física relacionada con la salud en niños y niñas de 10 a 12 años. Cuando los niños fueron divididos en base a su condición física cardiovascular, los resultados mostraron mejores valores para los estudiantes con alta condición física en el IMC, pliegues cutáneos, porcentaje de grasa corporal, salto de longitud, Course Navette y consumo máximo de oxígeno estimado. En cambio, la fuerza medida mediante la dinamometría manual no mostró diferencias estadísticamente significativas.

En este mismo sentido, Ortega et al. (2005) en su estudio realizado con adolescentes $(n=2.859,13-18,5$ años) encontraron que el grupo de jóvenes cuya capacidad aeróbica era superior a la indicativa de riesgo cardiovascular futuro poseían también un mayor rendimiento en las demás cualidades físicas (fuerza, dinamometría manual, salto de longitud, suspensión en barra; velocidad/agilidad, carrera de ida y vuelta 4 x $10 \mathrm{~m}$; flexibilidad, sit-and-reach), excepto en la dinamometría manual en las mujeres y la flexibilidad en los varones. Casajús et al. (2006) en su investigación con niños y adolescentes ( $\mathrm{n}=2.869$ ) de 7-17 años encontraron que el grupo de jóvenes con una alta condición física cardiovascular tenía un mayor consumo máximo de oxígeno y un menor IMC y suma de pliegues cutáneos.

En cuanto al número de estudios de asociación entre los niveles de capacidad cardiorrespiratoria y la condición física en jóvenes se ha incrementado en los últimos años. Varios estudios han encontrado una asociación entre los niveles de condición física cardiovascular y otros componentes de la condición física como la composición corporal, fuerza muscular, flexibilidad, velocidad-agilidad y coordinación (Ara et al., 2007; Brunet et al., 2007). En estudios con niños $\mathrm{y}$ adolescentes mostraron que los jóvenes que presentaban altos niveles de condición física cardiovascular tenían una menor cantidad de grasa corporal total presente (Ara et al., 2004; Lee y Arslanian, 2007; Ruiz et al., 2006) y futura (Ara et al., 2006; Eisenmann, Wickel, Welk, y Blair, 2005). En estudios en los que la condición física cardiovascular era asociada con la grasa abdominal también se encontraron los mismos resultados (Hussey, Bell, Bennett, O’Dwyer, y Gormley, 2007; Lee y Arslanian, 2007; Ortega et al., 2007; Winsley, Armstrong, Middlebrooke, Ramos-Ibáñez, y Williams, 2006). Asimismo, incluso entre los niños 
que presentaban sobrepeso $\mathrm{u}$ obesidad, aquellos que tenían una mayor capacidad cardiorrespiratoria eran los que tenían unos menores niveles de grasa corporal (Nassis, Psarra, y Sidossis, 2005).

Además de la capacidad cardiorrespiratoria, en la actualidad otro índice definitorio de la condición física relacionada con la salud es la fuerza muscular. En estudios con adultos la dinamometría manual se ha revelado como otro potente predictor de mortalidad y esperanza de vida (Metter, Talbot, Schrager, y Conwit, 2002), si bien los mecanismos que determinan esta relación no están del todo claros (Castillo Garzón et al., 2005). En nuestro estudio no se encontró una asociación entre el estado de la capacidad cardiorrespiratoria y la fuerza de presión manual. En este mismo sentido, Ortega et al. (2005) no encontraron dicha influencia en la dinamometría manual entre las mujeres adolescentes, aunque sí cuando se analizaban los varones. Por ello, parece que la fuerza medida mediante la dinamometría manual no presenta una relación tan clara con la capacidad cardiorrespiratoria como con las otras pruebas utilizadas. Estos resultados podrían estar afectos por problemas metodológicos como, por ejemplo, que en los resultados de la resistencia cardiorrespiratoria medido con el Course Navette existe una influencia del estado del peso de los niños, mientras que no ocurre lo mismo cuando se mide la fuerza mus- cular mediante la dinamometría manual. Una limitación del presente estudio fue que debido al tamaño de la muestra no se pudo realizar análisis separados por géneros. Consecuentemente, futuros estudios deberían abordar la relación de presentar una alta (o baja) capacidad cardiorrespiratoria y los niveles de condición física relacionada con la salud en niños y niñas separadamente. Asimismo, se deberían administrar diferentes pruebas que ayuden a comprender mejor la relación con determinadas componentes de la condición física como, por ejemplo, la fuerza muscular.

\section{Conclusiones}

Los niños y niñas de 10 a 12 años con una alta condición física cardiovascular presentan menores valores de IMC, pliegues cutáneos (triceps y pierna medial), porcentaje de grasa corporal, así como mayores valores de salto de longitud, Course Navette, y consumo de oxígeno máximo que sus compañeros con una menor capacidad cardiorrespiratoria. Las pruebas incluidas en la batería de tests ALPHA basada en la evidencia parecen presentar una clara relación con la salud cardiovascular de los niños y niñas de 10-12 años, excepto para la dinamometría manual en la que no existe una relación tan clara.

\section{BIBLIOGRAFÍA}

Alvero Cruz, J. R., Cabañas Armesilla, M. D., Herrero de Lucas, A., Martínez Riaza, L., Moreno Pascual, C., Porta Manzañido, J., Sirvent Belando, J. E. (2009). Protocolo de valoración de la composición corporal para el reconocimiento médico-deportivo. Documento de consenso del grupo español de cineantropometría de la federación española de medicina del deporte. Archivos de Medicina del Deporte, 26(131), 166-179. Disponible en http://www.femede.es/page.php?/Publicaciones/RevistaAMD

Ara, I., Moreno, L. A., Leiva, M. T., Gutin, B., y Casajús, A. (2007). Adiposity, physical activity, and physical fitness among children from Aragón. Obesity, 15, 1918-1924. doi:10.1038/oby.2007.228

Ara, I., Vicente-Rodríguez, G., Jiménez-Ramírez, J., Dorado, C., SerranoSánchez, J. A., y Calbet, J. A. (2004). Regular participation in sports is associated with enhanced physical fitness and lower fat mass in prepubertal boys. International Journal of Obesity, 28, 1585-1593. doi:10.1038/sj.ijo.0802754

Ara, I., Vicente-Rodríguez, G., Pérez-Gómez, J., Jiménez-Ramírez, J., Serrano-Sánchez, J. A., Dorado, C., y Calbet, J. A. L. (2006). Influence of extracurricular sport activities on body composition and physical fitness in boys: A 3-year longitudinal study. International Journal of Obesity, 30, 1062-1071. doi:10.1038/sj.ijo.0803303

Ardoy, D. N., Fernández-Rodríguez, J. M., Chillón, P., Artero, E. G., España-Romero, V., Jiménez-Pavón, D., Ortega, F. B. (2010). Educando para mejorar el estado de forma física, estudio Edufit: Antecedentes, diseño, metodología y análisis del abandono/ adhesión al estudio. $R e-$ vista Española de Salud Pública, 84(2), 151-168. Disponible en www. scielosp.org

Asociación Médica Mundial (2008). Declaración de Helsinki de la Asociación Médica Mundial: Principios éticos para las investigaciones médicas en seres humanos. Disponible en http://www.wma.net/s/policy/b3.htm
Bertoli, A., Di Daniele, N., Ceccobelli, M., Ficara, A., Girasoli, C. y De Lorenzo, A. (2003). Lipid profile, BMI, body fat distribution, and aerobic fitness in men with metabolic syndrome. Acta Diabetologica, 40, 130S133S. doi:10.1007/s00592-003-0045-7

Brunet, M., Chaput, J. P., y Tremblay, A. (2007). The association between low physical fitness and high body mass index or waist circumference is increasing with age in children: The 'Quebec en Forme' Project. International Journal of Obesity, 31, 637-645. doi:10.1038/ sj.ijo.0803448

Casajús, J. A., Leiva, M. T., Ferrando, J. A., Moreno, L., Aragonés, M. T. y Ara, I. (2006). Relación entre la condición física cardiovascular y la distribución de grasa en niños y adolescentes. Apunts. Medicina de L'esport, 41(149), 7-14. Disponible en http://www.apunts.org

Castillo Garzón, M. J., Ortega Porcel, F. B. y Ruiz Ruiz, J. (2005). Mejora de la forma física como terapia antienvejecimiento. Medicina Clínica, 124, 146-155. doi:10.1157/13071011

De la Cruz Sánchez, E., y Pino Ortega, J. (2010). Análisis de la condición física en escolares extremeños asociada a las recomendaciones de práctica de actividad física vigentes en España. Cultura, Ciencia y Deporte, 5(13), 45-49. Disponible en http://www.ucam.edu/ccd

Eisenmann, J. C., Wickel, E. E., Welk, G. J., y Blair, S. N. (2005). Relationship between adolescent fitness and fatness and cardiovascular disease risk factors in adulthood: The Aerobics Center Longitudinal Study (ACLS). American Heart Journal, 149, 46-53. doi:10.1016/j. ahj.2004.07.016

España-Romero, V., Artero, E. G., Santaliestra-Pasias, A. M., Gutierrez, A., Castillo, M. J. y Ruiz, J. R. (2008). Hand span influences optimal grip span in boys and girls aged 6 to 12 years. Journal of Hand Surgery, 33, 378-384. doi:10.1016/j.jhsa.2007.11.013 
Evenson, K. R., Stevens, J., Cai, J., Thomas, R., y Thomas, O. (2003). The effect of cardiorespiratory fitness and obesity on cancer mortality in women and men. Medicine \& Science in Sports \& Exercise, 35, 270-277. doi:10.1249/01.MSS.0000053511.02356.72

Froberg, K., y Andersen, L. B. (2005). Mini review: Physical activity and fitness and its relations to cardiovascular disease risk factors in children. International Journal of Obesity, 29, S34-S39. doi:10.1038/ sj.ijo.0803096

González-Gross, M., Ruiz, J. R., Moreno, L. A., De Rufino-Rivas, P., Garaulet, M., Mesana, M. I., y Gutiérrez, A. (2003). Body composition and physical performance of Spanish adolescents: The AVENA pilot study. Acta Diabetólogica, 40, S299-301. doi:10.1007/s00592-0030092-0

Hussey, J., Bell, C., Bennett, K., O’Dwyer, J. y Gormley, J. (2007). Relationship between the intensity of physical activity, inactivity, cardiorespiratory fitness and body composition in 7-10-year-old Dublin children. British Journal of Sports Medicine, 41, 311-316. doi:10.1136/ bjsm.2006.032045

International Society for the Advancement of Kinanthropometry (2001). International standards for anthropometric assessment. Underdale, Australia: ISAK.

Kodama, S., Saito, K., Tanaka, S., Maki, M., Yachi, Y., Asumi, M.,... Sone, H. (2009). Cardiorespiratory fitness as a quantitative predictor of allcause mortality and cardiovascular events in healthy men and women: A meta-analysis. The Journal of the American Medical Association, 301, 2024-2035. doi:10.1001/jama.2009.681

Lakka, T. A., Laaksonen, D. E., Lakka, H. M., Mannikko, N., Niskanen, L. K., Rauramaa, R. y Salonen, J. T. (2003). Sedentary lifestyle, poor cardiorespiratory fitness, and the metabolic syndrome. Medicine \& Science in Sports \& Exercise, 35, 1279-1286. doi:10.1249/01. MSS.0000079076.74931.9

Lee, S. J. y Arslanian, S. A. (2007). Cardiorespiratory fitness and abdominal adiposity in youth. European Journal of Clinical Nutrition, 61, 561565. doi:10.1038/sj.ejcn.1602541

Lee, C. D. y Blair, S. N. (2002). Cardiorespiratory fitness and smoking-related and total cancer mortality in men. Medicine \& Science in Sports \& Exercise, 34, 735-739. Disponible en http://journals.lww.com/acsmmsse/pages/default.aspx

Léger, L. A., Mercier, D., Gadoury, C. y Lambert, J. (1988). The multistage $20 \mathrm{~m}$ shuttle run test for aerobic fitness. Journal of Sports Science, 6(2), 93-101. Disponible en http://www.tandf.co.uk/journals/ rjsp

Metter, E. J., Talbot, L. A., Schrager, M. y Conwit, R. (2002). Skeletal muscle strength as a predictor of all-cause mortality in healthy men. Journal of Gerontology, 57(10), B359-365. Disponible en http:// biomedgerontology.oxfordjournals.org/

Myers, J., Prakash, M., Froelicher, V., Do, D., Partington, S. y Atwood, J. E. (2002). Exercise capacity and mortality among men referred for exercise testing. The New England Journal of Medicine, 346(11), 793-801. Disponible en www.nejm.org

Navarro, D., Fernández, J. M., Chillón, P., España-Romero, V., Artero, E. G., Jiménez-Pavón, D., Ortega, F. B. (2010). Efectos de un programa de educación física orientado a la mejora de la capacidad cardio-respiratoria en adolescentes (Estudio Edufit). Educando hacia el fitness. Cultura, Ciencia y Deporte, 5(13), S29. Disponible en http://www. ucam.edu/ccd

Nassis, G. P., Psarra, G., y Sidossis, L. S. (2005). Central and total adiposity are lower in overweight and obese children with high cardiorespiratory fitness. European Journal of Clinical Nutrition, 59, 137-141. doi:10.1038/sj.ejcn.1602061

Ortega, F. B., Ruiz, J. R., Castillo, M. J., Moreno, L. A., González-Gross, M., Warnberg, J. y Gutiérrez, A. (2005). Bajo nivel de forma física en los adolescentes españoles. Importancia para la salud cardiovascular futura (Estudio AVENA). Revista Española de Cardiología, 58, 898-909. doi:10.1157/13078126

Ortega, F. B., Ruiz, J. R., Castillo, M. J. y Sjöström, M. (2008). Physical fitness in childhood and adolescence: A powerful marker of health. International Journal of Obesity, 32, 1-11. doi:10.1038/sj.ijo.0803774

Ortega, F. B., Tresaco, B., Ruiz, J. R., Moreno, L. A., Martin-Matillas, M., Mesa, J. L., Castillo, M. J. (2007). Cardiorespiratory fitness and sedentary activities are associated with adiposity in adolescents. Obesity, 15, 1589-1599. doi:10.1038/oby.2007.188

Ramírez-Lechuga, J., Zabalaz Díaz, M., Sánchez-Muñoz, C., Som Castillo, A., Muros Molina, J. J. y Femia Marzo, P. (2010). Mejora de la capacidad aeróbica mediante un programa de entrenamiento de 8 semanas en adolescentes. Cultura, Ciencia y Deporte, 5(13), S17. Disponible en http://www.ucam.edu/ccd

Ruiz, J. R., Castro-Piñero, J., España-Romero, V., Artero, E. G., Ortega, F. B., Cuenca, M. M., Castillo, M. J. (2011). Field-based fitness assessment in young people: The ALPHA health-related fitness test battery for children and adolescents. British Journal of Sports Medicine, 45, 518-524. doi:10.1136/bjsm.2010.075341

Ruiz, J. R., España-Romero, V., Ortega, F. B., Sjöström, M., Castillo, M. J. y Gutiérrez, A. (2006). Hand span influences optimal grip span in male and female teenagers. The Journal of Hand Surgery, 31, 13671372. doi:10.1016/j.jhsa.2006.06.014

Ruiz, J. R., Rizzo, N. S., Hurtig-Wennlöf, A., Ortega, F. B., Warnberg, J. y Sjöström, M. (2006). Relations of total physical activity and intensity to fitness and fatness in children; The European Youth Heart Study. The American Journal of Clinical Nutrition, 84, 298-302. doi:10.3945/ ajcn.2008.27261.

Salinas Martínez, F., Miranda León, M. T. y Viciana Ramírez, J. (2006). La planificación de la educación física en su etapa de formación inicial. Estudio comparativo de los docentes de Murcia y España. Cultura, Ciencia y Deporte, 3(4), 3-12. Disponible en http://www.ucam.edu/ccd Sawada, S. S., Lee, I. M., Muto, T., Matuszaki, K. y Blair, S. N. (2003a). Cardiorespiratory fitness and the incidence of type 2 diabetes: Prospective study of Japanese men. Diabetes Care, 26(10), 2918-2922. Disponible en http://care.diabetesjournals.org/

Sawada, S. S., Muto, T., Tanaka, H., Lee, I. M., Paffenbarger, R. S., Shindo, M. y Blair, S. N. (2003b). Cardiorespiratory fitness and cancer mortality in Japanese men: A prospective study. Medicine \& Science in Sports \& Exercise, 35, 1546-1550. doi:10.1249/01.MSS.0000084525.06473.8E

Seibaek, M., Vestergaard, H., Burchardt, H., Sloth, C., Torp-Pedersen, C., Nielsen, S. L., Pedersen, O. (2003). Insulin resistance and maximal oxygen uptake. Clinical Cardiology, 26, 515-520. doi:10.1002/ clc. 4960261107

Slaughter, M. H., Lohman, T. G., Boileau, R. A., Horswill, C. A., Stillman, R. J., Van Loan, M. D. y Bemben, D. A. (1988). Skinfold equations for estimation of body fatness in children and youth. Human Biology, 60(5), 709-723. Disponible en http://www.humbiol.com/about-human-biology.html

Watanabe, T., Owashi, K., Kanauchi, Y., Mura, N., Takahara, M. y Ogino, T. (2005). The short-term reliability of grip strength measurement and the effects of posture and grip span. The Journal of Hand Surgery, 30, 603-609. doi:10.1016/j.jhsa.2004.12.007

Weir, J. P. (2005). Quantifying test-retest reliability using the intraclass correlation coefficient and the SEM. Journal of Strength and Conditioning Research, 19(1), 231-240. Disponible en http://journals.lww.com/ nsca-jscr/pages/default.aspx

Winsley, R. J., Armstrong, N., Middlebrooke, A. R., Ramos-Ibáñez, N., y Williams, C. A. (2006). Aerobic fitness and visceral adipose tissue in children. Acta Paediatrica, 95, 1435-1438. doi:10.1080/ 08035250600643244 